\title{
Quantum Non-Demolition Bell State Measurement and N-party GHZ State Preparation in Quantum Dot
}

\author{
Guo-Ping Guo*, Hui Zhang, and Guang-Can Guo \\ Key Laboratory of Quantum Information, University of Science and \\ Technology \\ of China, CAS, Hefei 230026, People's Republic of China
}

\begin{abstract}
By exploiting the fermionic qubit parity measurement, we present a scheme to realize quantum non-demolition (QND) measurement of Bell-states and generate n-party GHZ state in quantum dot. Compared with the original protocol, the required electron transfer before and after parity measurement can be nonadiabatic, which may speed up the operation speed and make the omitting of spin-orbit interaction more reasonable. This may help us to construct CNOT gate without highly precise control of coupling as the way of D. Gottesman and I. L. Chuang.
\end{abstract}

\section{INTRODUCTION}

Since D. Loss and D. P. DiVicenzo proposed a quantum computation protocol based on trapping spin electrons in semiconductor quantum dot (QD) in 1998, the potential of QDs for implementing tasks in quantum information processing (QIP) has been intensely studied both theoretically and experimentally [1,2]. The spin of an excess electron in the dot represents a promising qubit realization in such systems and it can be more accessible and scalable compared with other microscopic systems such as atoms or ions [3]. In work [4], DiVincenzo put forward five criteria, which must be all satisfied for any physical implementation of a quantum computer. There is a detailed review about the experimental progress on the spin qubit proposal using these five criteria [5]. It has been claimed that three criteria (well defined qubits, initialization and readout) have been already achieved and future experiments may focus on measuring the coherence time via the coherent manipulation of single spin and the coherent coupling via the manipulation of spins in neighboring dots.

Although it has been shown [6-8] that partial measurement is sufficient for quantum computation with photons, the extension of this paradigm to other systems is highly desirable and challenging. In the so-called measurementbased quantum computation, gates coupling qubits are no longer required. This eliminates the need of highly precise control of the strength and pulsing between qubits. Recently, H.-A. Engel and D. Loss presented a novel protocol of electrons' spin parity measurement [9], which explores the fact that resonant tunneling between the dots with different Zeeman splitting is only possible when the spins are antiparallel. By measuring the charge distributions between the two dots with a charge detector, such as quantum point contact (QPC) [10-12], one can figure out that two electrons initially loaded in one QD have either parallel or antiparallel spin configuration without demolition. In addition, they suggested a construction of CNOT gates in the way of C. W. J. Beenakker et. al [8], which requires two parity measurements, an ancillary qubit, a single-qubit measurement, and the application of single-qubit operations depending on the measurement outcomes. However, this CNOT construction requires that the electron transferring between two dots should be adiabatic to keep the electrons' spin states in each dot unchanged after a processing of loading the two dots electrons together and then separating them [13].

Consider two identical quantum dots 1 and 2 each with one electron initially in state $\left(a|\uparrow\rangle_{1}+b|\downarrow\rangle_{1}\right)$ and $\left(c|\uparrow\rangle_{2}+d|\downarrow\rangle\right.$ 2). The subscripts 1 and 2 depict the electrons in dot 1 and 2 respectively. The electron in dot 1 is transferred into dot 2 by operation, which acts on electron charge degree of freedom and keeps the electron spin state unchanged. Later, separate out one of the two electrons in dot 2 back into dot 1 . If these processes of transferring electron into and out of dot 2 are adiabatic, the two electrons in dot 1 and 2 can respectively remain in spin states $\left(a|\uparrow\rangle_{1}+b|\downarrow\rangle_{1}\right)$ and $\left(c|\uparrow\rangle_{2}+d|\downarrow\rangle_{2}\right)$ after these processes [14]. It is noted that the electron transfer between dots is made by acting on electron charge degree of freedom, which preserves electrons' spin state. If these electron transfer processes are nonadiabatic, the electron in dot 1 can also be transferred into dot 2 while its spin state is preserved. And the two electrons in dot 2 respectively in spin states $\left(a|\uparrow\rangle_{2}+b|\downarrow\rangle_{2}\right)$ and $\left(c|\uparrow\rangle_{2}+d|\downarrow\rangle_{2}\right)$ will have equal probability to be separated into dot 1 in these nonadiabatic transfer processes. Then there is $50 \%$ probability that the two electrons in dot 1 and 2 will remain in spin states

${ }^{*}$ Electronic address: gpguo@ustc.edu.cn 


$$
\left(a|\uparrow\rangle_{1}+b|\downarrow\rangle_{1}\right)\left(c|\uparrow\rangle_{2}+d|\downarrow\rangle_{2}\right)
$$

when one electron is transferred back into dot 1 with nonadiabatic processes. There is also $50 \%$ probability that the separated out electron into dot 1 initially stays at dot 2 and in spin state $\left(c|\uparrow\rangle_{2}+d|\downarrow\rangle_{2}\right)$. Then the two electrons in dot 1 and 2 will changed into spin states

$$
\left(c|\uparrow\rangle_{1}+d|\downarrow\rangle_{1}\right)\left(a|\uparrow\rangle_{2}+b|\downarrow\rangle_{2}\right) .
$$

In this case, it seems as if the two electrons in dot 1 and 2 have exchanged their spin states after all these processes. Although the nonadiabatic electron transfer process can be performed faster than the adiabatic one, we can not employ the C. W. J Beenakker et. al way [8] to construct CNOT gate if the two electrons after separating can be either state (1) or state (2) $[13,14]$.

Here we propose a scheme to realize Bell-state QND measurement and prepare n-party GHZ state with H.-A. Engel and D. Loss electron spin parity measurement. Compared with the original protocol, the present scheme emplores nonadiabatic electron transfer processes are emplored and there is uncertainty in separating the two electrons with different spins from one dot after the parity measurement. As single qubit rotations of electron in individual quantum dot can be straightly achieved with radio-frequency field, we can thus construct CNOT gate without highly precise controlled coupling by exploring the electron parity measurement in the way of D. Gottesman and I. L. Chuang $[9,15]$. As the electron transferring between dots, which is realized by acting on electron charge degree of freedom and preserves the electron spin, does not need to be adiabatic, the operations speeds may be enhanced and the neglecting of spin-orbital interaction may be more reasonable.

\section{THE BELL-STATE QND MEASUREMENT}

The idea of Bell-state QND measurement is firstly proposed by G.-P. Guo and C.-F.Li [16].If two qubits are initially in a Bell state, the measurement can check which Bell state they are in without destruction. And if the two qubits are not in Bell states, they can be prepared in any Bell state. In this sense, the QND measurement can be used as both a complete Bell-state analyzer and a Bell states generator. In the original QND protocol, CNOT gates are employed, which challenges its realization under the present experimental conditions. Now through the electron spin parity measurement of H.-A. Engel and D. Loss, the Bell states QND measurement can be implemented straightforwardly as shown in Fig 1, even although nonadiabatic electron transferring is emplored.

In the first step, two electrons 1 and 2 are both loaded into dot A. This electron transfer process can be made by acting on electron charge degree of freedom and then accordingly can preserve electron spin state. In this stage, the gate between the dots $\mathrm{A}$ and $\mathrm{B}$ is closed and the two electrons stay in $\operatorname{dot} \mathrm{A}$. The two quantum point contact(QND) charge detectors $D_{1}(0)=1$ and $D_{2}(0)=0$. In the second step, we open the gate for some time $t$ (about 20ns [9]). If their spins are antiparallel, the two electrons can tunnel to dot B. Then two charge detectors will click as $D_{1}(t)=0$ and $D_{2}(t)=1$. On the other hand, if their spin are parallel, the two electrons will stay in dot A and the detectors remains as $D_{1}(t)=1$ and $D_{2}(t)=0$. In this way, we know whether the two-electron spin state $|A A\rangle$ belongs to the Hilbert space of states $\left\{\Phi^{ \pm}=(|\uparrow \uparrow\rangle \pm|\downarrow \downarrow\rangle) / \sqrt{2}\right\}$ or $\left\{\Psi^{ \pm}=(|\uparrow \downarrow\rangle \pm|\downarrow \uparrow\rangle) / \sqrt{2}\right\}$. This is just the H.-A. Engel and D. Loss' original partial Bell-state measurement.

To realize Bell-state QND measurement, we proceed to separate the two electrons into two dots with some static field acting on electron charge degree of freedom. As the two electrons are separated in two dots, radio-frequency fields can address individual electron spin. We can preform single qubit operations and respectively rotate the two electrons spins as $|\uparrow\rangle \Rightarrow(|\uparrow\rangle+|\downarrow\rangle) / \sqrt{2},|\downarrow\rangle \Rightarrow(|\uparrow\rangle-|\downarrow\rangle) / \sqrt{2}$. Accordingly, these Hadamard operations will rotate the two-electron state as [16]:

$$
\hat{H}_{1} \hat{H}_{2}\left(\begin{array}{c}
\Phi^{+} \\
\Phi^{-} \\
\Psi^{+} \\
\Psi^{-}
\end{array}\right)=\left(\begin{array}{c}
\Phi^{+} \\
\Psi^{+} \\
\Phi^{-} \\
-\Psi^{-}
\end{array}\right) .
$$

Then we reload the two electrons into one $\operatorname{dot}($ for example $\operatorname{dot} \mathrm{A})$, and re-open the gate for some time $t$. We observe the two detectors $D_{1}(2 t)$ and $D_{2}(2 t)$. Combined with $D_{1}(t)$ and $D_{2}(t)$, we can determine exactly which Bell state the two electrons initially belong to just as shown in Table 1 .

Table 1: The states of the two detectors corresponding to each Bell state 


\begin{tabular}{|l|l|l|l|l|}
\hline & $\Psi^{+}$ & $\Psi^{-}$ & $\Phi^{+}$ & $\Phi^{-}$ \\
\hline$\left|D_{1}(t) D_{2}(t)\right\rangle$ & $01\rangle$ & $01\rangle$ & $10\rangle$ & $10\rangle$ \\
\hline \hline$\left.D_{1}(2 t) D_{2}(2 t)\right\rangle$ & $01\rangle$ & $10\rangle$ & $10\rangle$ & $01\rangle$ \\
\hline
\end{tabular}

Lastly, we separate the two electrons and irradiate them to perform Hadamard operations as the above steps. In this way, the final output state will recover to the initial state if the two electrons is originally in a Bell state and a complete Bell-state measurement is implemented without destruction.

As all operations are identical to the two electrons, we need not know which electron (with different spin state) is separated out from dot B in this Bell-state QND measurement. Distinguished with the original protocol, all the electron transfer processes between dots can thus be non-adiabatic and directly realized by acting on electron charge degree of freedom.

Obviously, if the two electrons are initially in an arbitrary state $\Phi_{12}=a \Phi^{+}+b \Phi^{-}+c \Psi^{+}+d \Psi^{-}$, where $a, b, c, d \in C$, the above measurement process will project them into one of the Bell states:

$$
\begin{aligned}
& \left(a \Phi^{+}+b \Phi^{-}+c \Psi^{+}+d \Psi^{-}\right) \otimes\left|D_{1}(t) D_{2}(t)\right\rangle \otimes\left|D_{1}(2 t) D_{2}(2 t)\right\rangle \\
\rightarrow & a \Phi^{+}|10\rangle \otimes|10\rangle+b \Phi^{-}|10\rangle \otimes|01\rangle+c \Psi^{+}|01\rangle \otimes|01\rangle+d \Psi^{-}|01\rangle \otimes|10\rangle .
\end{aligned}
$$

For example, we have a probability of $|d|^{2}$ to project the two electrons into state $\Psi^{-}$. With some single qubit rotations, we can thus get any other Bell-state. In this sense, the above measurement can be also regarded as spin-electron Bell states generation protocol, which theoretically has unit efficiency.

Actually, there is no need for two charge detectors, as the signal from D1 is always anti-correlated with the signal from D2. Therefore a single charge detector is sufficient. This would simplify the experimental setup.

\section{PREPARATION OF THE N-PARTICLE GHZ STATE}

After the demonstration of two electrons full Bell states measurements, we now discuss the preparation of the prior required GHZ entanglement states for the CNOT gate construction [15]. We assume that we have gotten two electrons $i, j$ in the state $\Phi_{i j}^{+}=(|\uparrow \uparrow\rangle+|\downarrow \downarrow\rangle) / \sqrt{2}$ in dot B from the above Bell-state QND measurement. Then we can separate one electron from dot B to dot A by static fields, which preserve their total spin-state $\Phi^{+}$[20]. Adjusting the bias voltage and the gate voltage between $\operatorname{dot} \mathrm{B}$ and $\operatorname{dot} \mathrm{C}$, we can load another electron $k$ from $\operatorname{dot} \mathrm{C}$ to $\operatorname{dot} \mathrm{B}$ as shown in Fig.2. The initial state of the electron in $\operatorname{dot} \mathrm{C}$ is $\Psi_{k}=\frac{1}{\sqrt{2}}\left(|\uparrow\rangle_{k}+|\downarrow\rangle_{k}\right)$. Thus the three-electron state can be written as (only the electron spin wavefunctions are shown):

$$
\begin{aligned}
\Psi_{i j k} & =\Phi_{i j}^{+} \otimes \Psi_{k}=\frac{1}{\sqrt{2}}\left(|\uparrow \uparrow\rangle_{i j}+|\downarrow \downarrow\rangle_{i j}\right) \otimes \frac{1}{\sqrt{2}}\left(|\uparrow\rangle_{k}+|\downarrow\rangle_{k}\right) \\
& =\frac{1}{2}\left(|\uparrow\rangle_{i}|\uparrow \uparrow\rangle_{j k}+|\downarrow\rangle_{i}|\downarrow \downarrow\rangle_{j k}+|\uparrow\rangle_{i}|\uparrow \downarrow\rangle_{j k}+|\downarrow\rangle_{i}|\downarrow \uparrow\rangle_{j k}\right) .
\end{aligned}
$$

The two electrons (electron 2 and 3 ) in dot B have $1 / 2$ probabilities to be measured in parallel spins to get state $\left(|\uparrow\rangle_{i}|\uparrow \uparrow\rangle_{j k}+|\downarrow\rangle_{i}|\downarrow \downarrow\rangle_{j k}\right) / \sqrt{2}$. In this case, when we separate one electron from dot B to dot C, the three electrons ( $i, j, k$ named the three electrons respectively in $\operatorname{dot} \mathrm{A}, \mathrm{B}$ and C) are in the state $\left(|\uparrow\rangle_{i}|\uparrow\rangle_{j}|\uparrow\rangle_{k}+|\downarrow\rangle_{i}|\downarrow\rangle_{j}|\downarrow\rangle_{k}\right) / \sqrt{2}$, which can be rotated into any other GHZ state with single qubit operation. Without doubt, the two electrons $j, k$ in $\operatorname{dot} \mathrm{B}$ have $1 / 2$ probabilities to be measured in anti-parallel spins to get state $\left(|\uparrow\rangle_{i}|\uparrow \downarrow\rangle_{j k}+|\downarrow\rangle_{i}|\downarrow \uparrow\rangle_{j k}\right) / \sqrt{2}$. In view of the indistinguishability of two electrons $j$ and $k$ in dot B and the uncertainty in separating them, we then have equal probability to get the state $\left(|\uparrow\rangle_{i}|\uparrow\rangle_{j}|\downarrow\rangle_{k}+|\downarrow\rangle_{i}|\downarrow\rangle_{j}|\uparrow\rangle_{k}\right) / \sqrt{2}$ and $\left(|\uparrow\rangle_{i}|\downarrow\rangle_{j}|\uparrow\rangle_{k}+|\downarrow\rangle_{i}|\uparrow\rangle_{j}|\downarrow\rangle_{k}\right) / \sqrt{2}$, when we separate one electron from dot B to dot $\mathrm{C}$. In this case, we can then reload the two electrons $i$ and $j$ into one dot and measure their parity again. After separating them, we then again have 1/2 probabilities to get the three electrons GHZ state $\left(|\uparrow\rangle_{i}|\uparrow\rangle_{j}|\downarrow\rangle_{k}+|\downarrow\rangle_{i}|\downarrow\rangle_{j}|\uparrow\rangle_{k}\right) / \sqrt{2}$. Two electrons $i$ and $j$ also have $1 / 2$ probabilities to be measured in antiparallel spins to get state $\left(|\uparrow \downarrow\rangle_{i j}|\uparrow\rangle_{k}+|\downarrow \uparrow\rangle_{i j}|\downarrow\rangle_{k}\right) / \sqrt{2}$. In this case, when we separate electrons $i, j$, we can have again equal probability to get the state $\left(|\uparrow\rangle_{i}|\downarrow\rangle_{j}|\uparrow\rangle_{k}+|\downarrow\rangle_{i}|\uparrow\rangle_{j}|\downarrow\rangle_{k}\right) / \sqrt{2}$ and $\left(|\downarrow\rangle_{i}|\uparrow\rangle_{j}|\uparrow\rangle_{k}+|\uparrow\rangle_{i}|\downarrow\rangle_{j}|\downarrow\rangle_{k}\right) / \sqrt{2}$. If necessary, we can again measure the spin parity of electron $j$ and $k$. In the process of repeating comparing the three electrons' spin, we can have a success probability $p$ of $1-\frac{1}{2^{m}}$ to get the electron GHZ state, where $m$ represents the times of comparing the electrons spins. When $m$ is large enough, $p \rightarrow 1$ and we can almost prepare three electrons GHZ state. Of course, if we only compare the spins of the two electrons $j, k$ initially in dot B one time, we have $1 / 2$ probabilities to get the GHZ state $\left(|\uparrow\rangle_{i}|\uparrow\rangle_{j}|\uparrow\rangle_{k}+|\downarrow\rangle_{i}|\downarrow\rangle_{j}|\downarrow\rangle_{k}\right) / \sqrt{2}$. 
Obviously, the above process can be directly performed on the case of n-electron GHZ states preparation $(n>3)$ by comparing the spins of the electrons between two dots in turn. In this way, the generation efficiency is $p^{n-2}$, where $p$ is the success probability of get three electron GHZ states. In fact, the above GHZ state generation procedure is very similar to the idea of S. Bose et. al. [22], which shows that the entangled states involving higher number of particles can be generated from entangled states involving lower number of particles by employing the same procedure as entanglement swaps. The basic ingredient of the original paper [21,22] is a Bell state measuring device and some L-particle (particle of lower number $(n=3)$ ) entanglement states. However, it can be proved that the required lower numbers of particles entanglement states can be just 2-particle entangled states with the present non-demolition partial Bell state measurement. Firstly, we prepare two copies of entangled states of $n / 2$ ( for example, assume $n$ is even) electrons in the form $\Psi_{n / 2}=\left(|\uparrow \uparrow \uparrow \ldots \uparrow\rangle_{123 \ldots \frac{n}{2}}+|\downarrow \downarrow \downarrow \ldots \downarrow\rangle_{123 \ldots \frac{n}{2}}\right) / \sqrt{2}$. Secondly, we coherently draw one electron from each of the two copies into a dot. By checking these two electrons spins (if it is parallel), we can get the entangled state of $n$ electrons $\Psi_{n}=\left(|\uparrow \uparrow \uparrow \ldots \uparrow\rangle_{123 \ldots n}+|\downarrow \downarrow \downarrow \ldots \downarrow\rangle_{123 \ldots n}\right) / \sqrt{2}$ with $1 / 2$ probability. As the success probability of each time parity checking is $1 / 2$, this $n(n \geq 2)$ electrons preparation protocol has an efficiency of $p^{n / 2-1}$ ( $n$ is even) or $p^{(n+1) / 2-1}$ ( $\mathrm{n}$ is odd). As the $n$ electrons in $\Psi_{n}$ are respectively in $n$ quantum dots, we can rotate it into any other $n$-particle GHZ entangled states with single-qubit operations.

\section{CONCLUSION}

Here we propose a protocol to realize the QND measurement of Bell-state, prepare $n$-electron GHZ states and then construct CNOT gate by employing the novel partial Bell-state measurement of Fermionic qubits in the article [9]. Distinguished with the previous protocol [9], the electron transfer processes before and after the spin parity measurement can nonadiabatic, which may make the omitting of the spin-orbit interaction effects in electron transport more sensible and speed up the computation operations. Furthermore, the present modified protocol has similar robustness to the experimental noises, such as the effect of extra phases from the inhomogeneous Zeeman splitting, finite $\mathrm{J}$ and different tunnel couplings of singlet and triplet. It is noted that the precision of the charge detectors or the fidelity of QPC measurements, which greatly influence the success of the electrons spin parity checking and the present protocols, have been recently analyzed in detail [10].

\section{Acknowledgments}

This work is funded by the National Fundamental Research Program (2001CB309300), National Nature Science Foundation of China (10304017), the Innovation Funds from Chinese Academy of Sciences.

[1] D. Loss and D. P. DiVincenzo, Phys. Rev. A 57, 120 (1998).

[2] B. E. Kane Nature 393, 119-120 (1998).

[3] Jeroen Martijn Elzerman's PHD thesis, Electron spin and charge in semiconductor quantum dots, chapter 1. Delft University of Technology, The Netherlands (2004).

[4] D. P. DiVincenzo, Fortschr. Phys. 48, 771 (2000).

[5] Jeroen Martijn Elzerman's PHD thesis, Electron spin and charge in semiconductor quantum dots, chapter 9. Delft University of Technology, The Netherlands (2004).

[6] E. Knill, R. Laflamme, G. Milburn, Nature 409,46 (2001).

[7] M. A. Nielsen, I. L. Chuang, Quantum Computation and Quantum Information (Cambridge Univ. Press, New York, 2000).

[8] C. W. J. Beenakker, et.al. , Phys. Rev. Lett. 93, 020501 (2004).

[9] Hans-Andres Engel and Daniel Loss, Science 309, 22 July 2005, VOL 309.

[10] J. M. Elzerman, et al. Nature 430, 431-435 (2004).

[11] Petta et al. Science 309, 2180, 2005. M. Kroutvar et al., Nature 432, 81 (2004).

[12] M. Field et al., Phys. Rev. Lett. 70, 1311 (1993).

[13] In C. W. J. Beenakker et. al [8] original propose of constructing CNOT gate, the photon with state $\left(a|0\rangle_{u}+b|1\rangle_{u}\right)$ input from up-path determinately comes out from up-path and the photon with state $\left(c|0\rangle_{d}+d|1\rangle_{d}\right)$ input from downpath determinately comes out from down-path after parity measurement. These two output photons will then undergo different operations. If the output photons from parity measurement circuit up-path has equal probability to be in either state $\left(a|0\rangle_{u}+b|1\rangle_{u}\right)$ or $\left(c|0\rangle_{u}+d|1\rangle_{u}\right)$, (the output photon from down-path correspondingly will be in either state $\left(c|0\rangle_{d}+d|1\rangle_{d}\right)$ or $\left.\left(a|0\rangle_{d}+b|1\rangle_{d}\right)\right)$, we then cannot determinate the following operations.

[14] Hans-Andres Engel and Daniel Loss, Science 309, 22 July 2005, VOL 309. 
[15] D. Gottesman and I. L. Chuang, Nature 402, 390 (1999).

[16] G.-P. Guo, C.-F. Li ,Phys. Lett. A 286, 401 (2001).

[17] H. -A. Engel et al., Phys. Rev. Lett. 93, 106804 (2004).

[18] W. Lu , Z. Ji , L. Pfeiffer , K. W. West, A. J. Rimberg, Nature 423 , 422 (2003).

[19] R. Schleser et al., Appl. Phys. Lett. 85 , 2005 (2004).

[20] W. G. van der Wiel, et al. Reviews of Modern Physics, VOL. 75, JANUARY 2003.

[21] G. P. Guo, et. al Phys. Rev. A 65, 042102 (2002).

[22] S. Bose, V. Vedral, and P. L. Knight, Phys. Rev. A 57, 822 (1998).

Fig. 1. Schematic picture of the Bell-state analyzer, which includes two coupled quantum dots (circle A and B), two quantum point contact charge detectors (triangle $\mathrm{D}_{1}$ and $\mathrm{D}_{2}$ ) and a gate (solid vertical line). Dot $\mathrm{A}$ and $\mathrm{B}$ are assumed to have different Zeeman splitting, and the individual tunneling events can be efficiently identified with a time resolved measurement [10,17-19]. The gate switches on and off the coupling between dot A and $\mathrm{B}$. Here we consider only the case that the two electrons are both in $\operatorname{dot} \mathrm{A}$ or in $\operatorname{dot} \mathrm{B}|A A\rangle$ and $|B B\rangle$, as the coupling to the state $|A B\rangle$ is small [9]. The two electrons tunneling between states $|A A\rangle$ and $|B B\rangle$ is only resonant, when they have antiparallel spins and in the Hilbert space $\left\{\Psi^{ \pm}=(|\uparrow \downarrow\rangle \pm|\downarrow \uparrow\rangle) / \sqrt{2}\right\}$. If they are in space $\left\{\Phi^{ \pm}=(|\uparrow \uparrow\rangle \pm|\downarrow \downarrow\rangle) / \sqrt{2}\right\}$, these two electrons will remain on the initial dot. This requirement is the key principle of electrons spin parity measurements. If the two electrons are both in the dot A (or in the dot B), $D_{1}=1$ and $D_{2}=0$ (or $D_{2}=1$ and $D_{1}=0$ ).

Fig. 2. Schematic picture for preparation of the 3-electron GHZ states. Initially, dot $\mathrm{A}, \mathrm{B}$ and $\mathrm{C}$ each has one electron. The electron transfer between quantum dots is required to preserve electron spin state, which can be achieved by static electrical fields acting on the electron charge degree of freedom. Spin parity measurement of two electrons is made in dot B. 


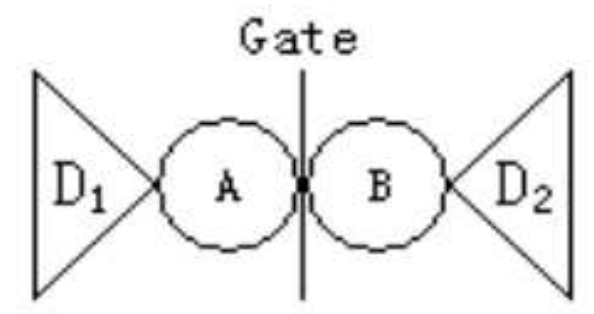

Fig. 1 


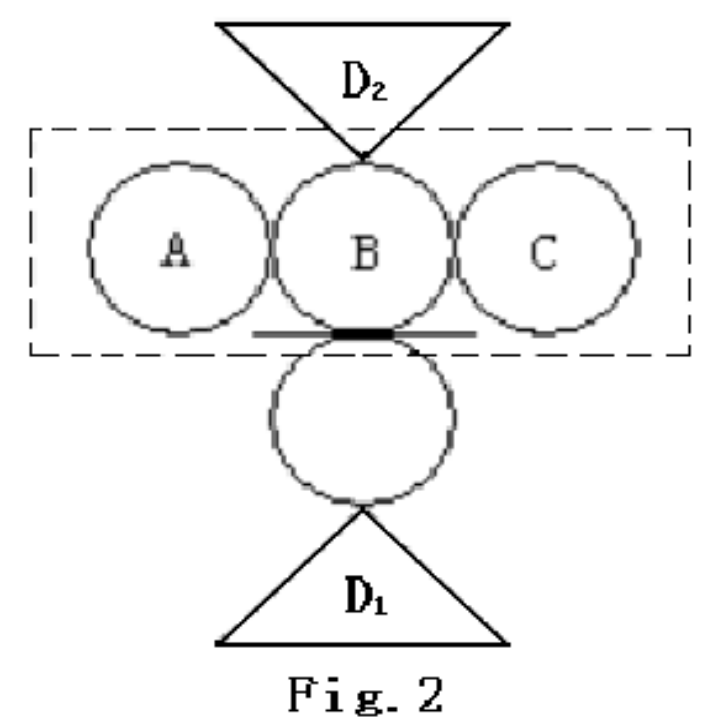

